\title{
On Refined Neutrosophic Algebraic Hyperstructures I
}

\author{
${ }^{1}$ A.A.A. Agboola , ${ }^{2}$ M.A. Ibrahim, ${ }^{3}$ E.O. Adeleke, ${ }^{4}$ S.A. Akinleye \\ $1,2,3,4$ Department of Mathematics, Federal University of Agriculture, Abeokuta, Nigeria. \\ agboolaaaa@funaab.edu.ng ${ }^{1}$, muritalaibrahim40@gmail.com ${ }^{2}$, yemi376@yahoo.com ${ }^{3}$, \\ sa_akinleye@yahoo.com ${ }^{4}$
}

\begin{abstract}
Given any algebraic hyperstructure $(X, *, \circ)$, the objective of this paper is to generate a refined neutrosophic algebraic hyperstructure $\left(X\left(I_{1}, I_{2}\right), *^{\prime}, o^{\prime}\right)$ from $X, I_{1}$ and $I_{2}$ and study refined neutrosophic Krasner hyperrings in particular.
\end{abstract}

Keywords: refined neutrosophic group, refined neutrosophic ring, refined neutrosophic hyperring.

\section{Introduction}

The concept of refined neutrosophic algebraic structures was introduced by Agboola in $!^{11}$ Adeleke et.al in ${ }^{24 \mid 25}$ introduced and studied refined neutrosophic rings and ideals. In the present paper, we introduce the concept of refined neutrosophic algebraic hyperstructure and we study refined neutrosophic Krasner hyperrings in particular. In this section, we present introduction and some necessary definitions for completeness.

Definition 1.1 $(22)$. A hypergroup $(X, \circ)$ is called a canonical hypergroup if the following conditions are satisfied:

(i) $(X, \circ)$ is commutative.

(ii) $(X, \circ)$ has a scalar identity that is $\forall x \in X$, there exists $e \in X$ such that

$$
x \circ e=e \circ x=x .
$$

(iii) Every element of $(X, \circ)$ has a unique inverse that is $\forall x \in X$, there exists a unique $x^{-1} \in X$ such that

$$
e \in x \circ x^{-1} \cap x^{-1} \circ x .
$$

(iv) $(X, \circ)$ is reversible that is if $x \in y \circ z$, then there exist $y^{-1}, z^{-1} \in X$ such that $z \in y^{-1} \circ x$ and $y \in x \circ z^{-1}$.

Definition 1.2 (22). An algebraic hyperstructure $(X,+,$.$) where +$ is a hyperoperation and. is the usual multiplication operation is called a Krasner hyperring if the following conditions are satisfied:

(i) $(X,+)$ is a canonical hypergroup with identity 0 .

(ii) $(X,$.$) is a semigroup with 0$ as a bilateral absorbing element that is

$$
x .0=0 . x=0 \quad \forall x \in X .
$$

(iii) . is distributive over + .

A Krasner hyperring $(X,+,$.$) is said to be commutative with unit element if (X,$.$) is a commutative semigroup$ with unit element.

Definition 1.3 (22). (i) Let $(X,+,$.$) be a Krasner hyperring and let A$ be a nonempty subset of $X . A$ is called a subhyperring of $X$ if $(A,+,$.$) is a hyperring in its own right.$ 
(ii) $A$ is called a normal subhyperring of $X$ if and only if $x+A-x \subseteq A$ for all $x \in X$.

(iii) $A$ is called a hyperideal of $X$ if for all $x \in X, a \in A$, we have $a . x, x . a \in A$.

Definition $1.4\left(\frac{22}{2}\right)$. Let $\left(X_{1},+,.\right)$ and $\left(X_{2},+^{\prime}, .^{\prime}\right)$ be two Krasner hyperrings. The mapping $\phi: X_{1} \rightarrow X_{2}$ is called a $\operatorname{good}\left(\right.$ strong) homomorphism if $\forall x, y \in X_{1}$, we have the following:

(i) $\phi(x+y)=\phi(x)+{ }^{\prime} \phi(y)$ and

(ii) $\phi(x . y)=\phi(x) .^{\prime} \phi(y)$.

The kernel of $\phi$ denoted by $\operatorname{Ker} \phi=\left\{x \in X_{1}: \phi(x)=0\right\}$.

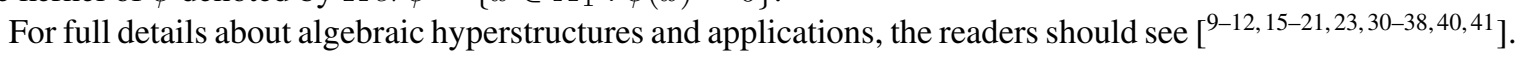

The concept of neutrosophic logic was introduced by Florentin Smarandache in 1995. Neutrosophic logic is an extension and generalization of fuzzy logic and intuitionistic fuzzy logic of Lofti Zadeh and Atanassov respectively. In neutrosophic logic, each proposition is approximated to have the percentage of truth in the subset $T$, percentage of indeterminacy in the subset $I$ and the percentage of falsity in the subset $F$ where $T, I, F \subseteq]^{-} 0,1^{+}$. For simplicity and practical applications, $T, I, F$ are taken as single-valued numbers from the interval $[0,1]$ with $0 \leq T+I+F \leq 3$. In neutrosophy, if $\langle A\rangle$ is an item or a concept, then only the triads $(<A>,<$ neut $A>,<$ anti $A>$ ) that makes sense in the real world. The neutrosophication of a concept into a $(T, I, F)$-concept, or more generally into a refined $\left(T_{1}, T_{2}, \cdots, I_{1}, I_{2}, \cdots, F_{1}, F_{2}, \cdots\right)$-concept is possible if the triad $(<$ concept $>,<$ neutconcept $>,<$ anticoncept $>$, or more generally its corresponding refined concept triad, makes sense in the real world. If the indeterminacy factor $I$ is refined into two indeterminacies $I_{1}$ and $I_{2}$ in the sense of Smarandache in, ${ }^{27}$ then we can take $I_{1}=$ contradiction (true $(T)$ and false $(F)$ ), $I_{2}=$ ignorance (true $(T)$ or false $(F)$ ) so that logically we have

$$
I_{1}^{2}=I_{1}, I_{2}^{2}=I_{2}, I_{1} I_{2}=I_{2} I_{1}=I_{1} .
$$

For full details about neutrosophy, neutrosphic logic and neutrosophic algebraic structures, the readers should see [26 $[28,39]$

Definition $1.5(24)$. Let $(R,+,$.$) be any ring. The abstract system \left(R\left(I_{1}, I_{2}\right),+,.\right)$ is called a refined neutrosophic ring generated by $R, I_{1}, I_{2} \cdot\left(R\left(I_{1}, I_{2}\right),+,.\right)$ is called a commutative refined neutrosophic ring if for all $x, y \in R\left(I_{1}, I_{2}\right)$, we have $x y=y x$. If there exists an element $e=(1,0,0) \in R\left(I_{1}, I_{2}\right)$ such that $e x=x e=x$ for all $x \in R\left(I_{1}, I_{2}\right)$, then we say that $\left(R\left(I_{1}, I_{2}\right),+,.\right)$ is a refined neutrosophic ring with unity.

Definition 1.6 $\left(^{(24}\right)$. Let $\left(R\left(I_{1}, I_{2}\right),+,.\right)$ be a refined neutrosophic ring and let $J\left(I_{1}, I_{2}\right)$ be a nonempty subset of $R\left(I_{1}, I_{2}\right) . J\left(I_{1}, I_{2}\right)$ is called a refined neutrosophic subring of $R\left(I_{1}, I_{2}\right)$ if $\left(J\left(I_{1}, I_{2}\right),+,.\right)$ is itself a refined neutrosophic ring. It is essential that $J\left(I_{1}, I_{2}\right)$ contains a proper subset which is a ring. Otherwise, $J\left(I_{1}, I_{2}\right)$ will be called a pseudo refined neutrosophic subring of $R\left(I_{1}, I_{2}\right)$.

Definition $1.7\left(\frac{25}{}\right)$. Let $R\left(I_{1}, I_{2}\right)$ be a refined neutrosophic ring and let $J$ be a nonempty subset of $R\left(I_{1}, I_{2}\right)$. $J$ is called a refined neutrosophic ideal of $R\left(I_{1}, I_{2}\right)$ if the following conditions hold:

(i) $J$ is a refined neutrosophic subring of $R\left(I_{1}, I_{2}\right)$.

(ii) For every $x \in J$ and $r \in R\left(I_{1}, I_{2}\right)$, we have $x r, r x \in J$.

If $J$ is a pseudo refined neutrosophic subring of $R\left(I_{1}, I_{2}\right)$ and for every $x \in J$ and $r \in R\left(I_{1}, I_{2}\right)$, we have $x r \in J$, then $J$ is called a pseudo refined neutrosophic ideal of $R\left(I_{1}, I_{2}\right)$.

Definition $1.8\left({ }^{25}\right)$. Let $J$ be a refined neutrosophic ideal of the refined neutrosophic ring $R\left(I_{1}, I_{2}\right)$. The set $R\left(I_{1}, I_{2}\right) / J$ is defined by

$$
R\left(I_{1}, I_{2}\right) / J=\left\{r+J: r \in R\left(I_{1}, I_{2}\right)\right\} .
$$

If $\bar{x}=r_{1}+J$ and $\bar{y}=r_{2}+J$ are two arbitrary elements of $R\left(I_{1}, I_{2}\right) / J$ and $\oplus, \odot$ are two binary operations on $R\left(I_{1}, I_{2}\right) / J$ defined by

$$
\begin{aligned}
& \bar{x} \oplus \bar{y}=(x+y)+J, \\
& \bar{x} \odot \bar{y}=(x y)+J .
\end{aligned}
$$

It can be shown that $\left(R\left(I_{1}, I_{2}\right) / J, \oplus, \odot\right)$ is a refined neutrosophic ring with the additive identity $J$. $\left(R\left(I_{1}, I_{2}\right) / J, \oplus, \odot\right)$ is called a refined quotient neutrosophic ring. 
Definition 1.9 $(25)$. Let $\left(R\left(I_{1}, I_{2}\right),+,.\right)$ and and $\left(S\left(I_{1}, I_{2}\right),+,.\right)$ be two refined neutrosophic rings. The mapping $\phi:\left(R\left(I_{1}, I_{2}\right),+,.\right) \rightarrow\left(S\left(I_{1}, I_{2}\right),+,.\right)$ is called a refined neutrosophic ring homomorphism if the following conditions hold:

(i) $\phi(x+y)=\phi(x)+\phi(y)$.

(ii) $\phi(x . y)=\phi(x) \cdot \phi(y)$.

(iii) $\phi\left(I_{k}\right)=I_{k} \forall x, y \in R\left(I_{1}, I_{2}\right)$ and $k=1,2$.

The image of $\phi$ denoted by $\operatorname{Im} \phi$ is defined by the set

$$
\operatorname{Im} \phi=\left\{y \in S\left(I_{1}, I_{2}\right): y=\phi(x) \text { for some } x \in R\left(I_{1}, I_{2}\right)\right\} .
$$

The kernel of $\phi$ denoted by $\operatorname{Ker} \phi$ is defined by the set

$$
\operatorname{Ker} \phi=\left\{x \in R\left(I_{1}, I_{2}\right): \phi(x)=(0,0,0)\right\} .
$$

Epimorphism, monomorphism, isomorphism, endomorphism and automorphism of $\phi$ are similarly defined as in the classical cases. For full details about neutrosophic and refined neutrosophic algebraic structures and algebraic hyperstructures, the readers should see $[1 / 8|13| 14|24| 25 \mid 29]$

\section{Development of Refined Neutrosophic Algebraic Hyperstructures}

In this section, we present the development of refined neutrosophic algebraic hyperstructures. Refined neutrosophic Krasner hyperrings are studied in particular and some of their basic properties are presented.

Suppose that $(X, *, \circ)$ is any given algebraic hyperstructure with $*$ as a hyperoperation on $X$ and $\circ$ is an operation on $X$. Let $X\left(I_{1}, I_{2}\right)=<X, I_{1}, I_{2}>$ be a refined neutrosophic set generated by $X, I_{1}, I_{2}$ defined by

$$
X\left(I_{1}, I_{2}\right)=\left\{\left(x, y I_{1}, z I_{2}\right): x, y, z \in X\right\} .
$$

Suppose that

$$
*^{\prime}: X\left(I_{1}, I_{2}\right) \times X\left(I_{1}, I_{2}\right) \rightarrow 2^{X\left(I_{1}, I_{2}\right)} \quad \text { and } \quad \circ^{\prime}: X\left(I_{1}, I_{2}\right) \times X\left(I_{1}, I_{2}\right) \rightarrow X\left(I_{1}, I_{2}\right)
$$

are respectively a hyperoperation and an operation on $X\left(I_{1}, I_{2}\right)$ and such that $\forall\left(a, b I_{1}, c I_{2}\right),\left(d, e I_{1}, f I_{2}\right) \in$ $X\left(I_{1}, I_{2}\right)$ with $a, b, c, d, e, f \in X$, we define

$$
\begin{aligned}
\left(a, b I_{1}, c I_{2}\right) *^{\prime}\left(d, e I_{1}, f I_{2}\right)= & \left\{\left(\left(x, y I_{1}, z I_{2}\right): x \in a * d, y \in(a * e)\right.\right. \\
& *(b * d) *(b * e) *(b * f) *(c * e), \\
& z \in(a * f) *(c * d) *(c * f)\} \\
\left(a, b I_{1}, c I_{2}\right) \circ^{\prime}\left(d, e I_{1}, f I_{2}\right)= & \left(\left(x, y I_{1}, z I_{2}\right)\right. \text { such that } \\
& x=a \circ d, y=(a \circ e) \circ(b \circ d) \\
& \circ(b \circ e) \circ(b \circ f) \circ(c \circ e), \\
& z=(a \circ f) \circ(c \circ d) \circ(c \circ f) .
\end{aligned}
$$

With the composition of the elements of $X\left(I_{1}, I_{2}\right)$ according to equations (1) and (2), we call the algebraic structure $\left(X\left(I_{1}, I_{2}\right), *^{\prime}, \circ^{\prime}\right)$ a refined neutrosophic algebraic hyperstructure. $\left(X\left(I_{1}, I_{2}\right), *^{\prime}, \circ^{\prime}\right)$ is named according to the algebraic laws satisfied by $*^{\prime}$ and $o^{\prime}$.

If $\left(X\left(I_{1}, I_{2}\right), *^{\prime}, \circ^{\prime}\right)$ and $\left(Y\left(I_{1}, I_{2}\right), *^{\prime \prime}, \circ^{\prime \prime}\right)$ are two given refined neutrosophic algebraic hyperstructures, then the mapping $\phi:\left(X\left(I_{1}, I_{2}\right), *^{\prime}, o^{\prime}\right) \rightarrow\left(Y\left(I_{1}, I_{2}\right), *^{\prime \prime}, o^{\prime \prime}\right)$ is called a refined neutrosophic good(strong) homomorphism if

$\forall\left(a, b I_{1}, c I_{2}\right),\left(d, e I_{1}, f I_{2}\right) \in X\left(I_{1}, I_{2}\right)$, the following conditions hold:

(i) $\left.\phi\left(\left(a, b I_{1}, c I_{2}\right) *^{\prime}\left(d, e I_{1}, f I_{2}\right)\right)=\phi\left(\left(a, b I_{1}, c I_{2}\right)\right) *^{\prime \prime} \phi\left(\left(d, e I_{1}, f I_{2}\right)\right)\right)$,

(ii) $\left.\phi\left(\left(a, b I_{1}, c I_{2}\right) \circ^{\prime}\left(d, e I_{1}, f I_{2}\right)\right)=\phi\left(\left(a, b I_{1}, c I_{2}\right)\right) \circ^{\prime \prime} \phi\left(\left(d, e I_{1}, f I_{2}\right)\right)\right)$,

(iii) $\phi\left(I_{i}\right)=I_{i}$ for $i=1,2$.

In all that follows in the sequel, all hyperrings $(X,+,$.$) will be Krasner hyperrings.$ 
Definition 2.1. Let $(X,+,$.$) be any hyperring and let X\left(I_{1}, I_{2}\right)=<X, I_{1}, I_{2}>$ be a set generated by $X, I_{1}$ and $I_{2}$. The triple $\left(X\left(I_{1}, I_{2}\right),+,.\right)$ is called a refined neutrosophic hyperring. It is said to be commutative if for all $x, y \in X\left(I_{1}, I_{2}\right)$, we have $x . y=y . x$.

For all $\left(a, b I_{1}, c I_{2}\right),\left(d, e I_{1}, f I_{2}\right) \in X\left(I_{1}, I_{2}\right)$, we define the composition of elements with respect to the hyperoperation + and ordinary operation . in $X\left(I_{1}, I_{2}\right)$ as follows.

$$
\begin{aligned}
\left(a, b I_{1}, c I_{2}\right)+\left(d, e I_{1}, f I_{2}\right)= & \left\{\left(x, y I_{1}, z I_{2}\right): x \in a+d, y \in b+e,\right. \\
& z \in c+f\} . \\
\left(a, b I_{1}, c I_{2}\right) \cdot\left(d, e I_{1}, f I_{2}\right)= & \left(x, y I_{1}, z I_{2}\right) \text { such that } x=a d, \\
& y \in(a e+b d+b e+b f+c e), \\
& z \in(a f+c d+c f) .
\end{aligned}
$$

Definition 2.2. (i) Let $\left(X\left(I_{1}, I_{2}\right),+,.\right)$ be a refined neutrosophic hyperring and let $A\left(I_{1}, I_{2}\right)$ be a nonempty subset of $X\left(I_{1}, I_{2}\right)$. Then $A\left(I_{1}, I_{2}\right)$ is called a refined neutrosophic subhyperring of $X\left(I_{1}, I_{2}\right)$ if $\left(A\left(I_{1}, I_{2}\right),+,.\right)$ is a refined neutrosophic hyperring in its own right. $-A\left(I_{1}, I_{2}\right)$ is defined as

$$
-A\left(I_{1}, I_{2}\right)=\left\{-a: a \in A\left(I_{1}, I_{2}\right)\right\}
$$

(iii) $A\left(I_{1}, I_{2}\right)$ is called a refined neutrosophic hyperideal of $X\left(I_{1}, I_{2}\right)$ if for all $x \in X\left(I_{1}, I_{2}\right), a \in A\left(I_{1}, I_{2}\right)$, we have $a . x, x . a \in A\left(I_{1}, I_{2}\right)$.

Definition 2.3. Let $A\left(I_{1}, I_{2}\right)$ and $B\left(I_{1}, I_{2}\right)$ be two refined neutrosophic hyperideals of a refined neutrosophic hyperring $\left(X\left(I_{1}, I_{2}\right),+,.\right)$.

(i) The sum $A\left(I_{1}, I_{2}\right)+B\left(I_{1}, I_{2}\right)$ is defined by

$$
A\left(I_{1}, I_{2}\right)+B\left(I_{1}, I_{2}\right)=\left\{x: x \in a+b \text { for some } a \in A\left(I_{1}, I_{2}\right), b \in B\left(I_{1}, I_{2}\right)\right\} .
$$

(ii) The product $A\left(I_{1}, I_{2}\right) B\left(I_{1}, I_{2}\right)$ is defined by

$$
\left.A\left(I_{1}, I_{2}\right) B\left(I_{1}, I_{2}\right)=\left\{x: x \in \sum_{i}^{n} a_{i} b_{i}, a_{i} \in A\left(I_{1}, I_{2}\right), b_{i} \in B\left(I_{1}, I_{2}\right)\right\}, n \in \mathbb{Z}^{+}\right\} .
$$

Definition 2.4. Let $A_{1}\left(I_{1}, I_{2}\right), A_{2}\left(I_{1}, I_{2}\right), \cdots, A_{n}\left(I_{1}, I_{2}\right)$ be refined neutrosophic hyperideals of a refined neutrosophic hyperring $\left(X\left(I_{1}, I_{2}\right),+,.\right)$.

$$
\mathbb{A}\left(I_{1}, I_{2}\right)=A_{1}\left(I_{1}, I_{2}\right) \oplus A_{2}\left(I_{1}, I_{2}\right) \oplus \cdots \oplus A_{n}\left(I_{1}, I_{2}\right)=\oplus \sum_{i=1}^{n} A_{i}\left(I_{1}, I_{2}\right)
$$

is called a direct sum if for each element $x$ in $\mathbb{A}\left(I_{1}, I_{2}\right)$, we have

$$
x \in \sum_{i}^{n} a_{i} \text { (uniquely), with } a_{i} \in A_{i}\left(I_{1}, I_{2}\right) .
$$

Definition 2.5. Let $\left(X_{1}\left(I_{1}, I_{2}\right),+,.\right)$ and $\left.X_{2}\left(I_{1}, I_{2}\right),{ }^{\prime}{ }^{\prime},{ }^{\prime}\right)$ be two refined neutrosophic hyperrings. The mapping $\phi: X_{1}\left(I_{1}, I_{2}\right) \rightarrow X_{2}\left(I_{1}, I_{2}\right)$ is called a refined neutrosophic good(strong) homomorphism if $\forall x, y \in$ $X_{1}\left(I_{1}, I_{2}\right)$, we have the following:

(i) $\phi(x+y)=\phi(x)+^{\prime} \phi(y)$ and

(ii) $\phi(x . y)=\phi(x){ }^{\prime} \phi(y)$.

(iii) $\phi\left(I_{i}\right)=I_{i}$ for all $i=1,2$.

The kernel of $\phi$ denoted by $\operatorname{Ker} \phi=\left\{x \in X_{1}\left(I_{1}, I_{2}\right): \phi(x)=0\right\}$. The image of $\phi$ denoted by $\operatorname{Im} \phi=\{y \in$ $X_{2}\left(I_{1}, I_{2}\right): y=\phi(x)$ for some $\left.x \in X_{1}\left(I_{1}, I_{2}\right)\right\}$. 
Example 2.6. Let $\left(\mathbb{R}\left(I_{1}, I_{2}\right),+,.\right)$ be a refined neutrosophic ring of real numbers and let $J\left(I_{1}, I_{2}\right)$ be a refined neutrosophic ideal of $\mathbb{R}\left(I_{1}, I_{2}\right)$. Let $\mathbb{R}\left(I_{1}, I_{2}\right) / J\left(I_{1}, I_{2}\right)$ be a refined neutrosophic quotient ring defined by

$$
\mathbb{R}\left(I_{1}, I_{2}\right) / J\left(I_{1}, I_{2}\right)=\left\{x+J\left(I_{1}, I_{2}\right): x \in \mathbb{R}\left(I_{1}, I_{2}\right)\right\} .
$$

Let $\oplus$ and $\odot$ be additive hyperoperation and ordinary multiplicative operation respectively defined on $\mathbb{R}\left(I_{1}, I_{2}\right) / J\left(I_{1}, I_{2}\right)$ as follows:

$$
\begin{aligned}
\left(x+J\left(I_{1}, I_{2}\right)\right) \oplus\left(y+J\left(I_{1}, I_{2}\right)\right) & =\left\{z+J\left(I_{1}, I_{2}\right): z \in(x+y) J\left(I_{1}, I_{2}\right)\right\} \\
\left(x+J\left(I_{1}, I_{2}\right)\right) \odot\left(y+J\left(I_{1}, I_{2}\right)\right) & =x y+J\left(I_{1}, I_{2}\right) .
\end{aligned}
$$

Then $\left(\mathbb{R}\left(I_{1}, I_{2}\right) / J\left(I_{1}, I_{2}\right), \oplus, \odot\right)$ is a refined neutrosophic hyperring.

Theorem 2.7. Any refined neutrosophic hyperring is a hyperring.

Proof. Suppose that $\left(R\left(I_{1}, I_{2}\right),+,.\right)$ is a refined neutrosophic hyperring. It is clear that:

(1) $\left(R\left(I_{1}, I_{2}\right),+\right)$ is a canonical hypergroup.

(2) $\left(R\left(I_{1}, I_{2}\right),.\right)$ is a semigroup and $0=\left(0,0 I_{1}, 0 I_{2}\right)$ is a bilateral absorbing element.

(3) We establish the distributive laws. Let $x=\left(a, b I_{1}, c I_{2}\right), y=\left(d, e I_{1}, f I_{2}\right), z=\left(g, h I_{1}, i I_{2}\right)$ be arbitrary elements in $R\left(I_{1}, I_{2}\right)$ with $a, b, c, d, e, f, g, h, i \in R$.

$$
\begin{aligned}
x(y+z)= & \left\{\left(p, q I_{1}, r I_{2}\right): p \in a(d+g), q \in(a(e+h)+b(d+g)+b(e+h)\right. \\
& +b(f+i)+c(e+h)), r \in(a(f+i)+c(d+g)+c(f+i))\}, \text { and } \\
x y+x z= & \left\{\left(u, v I_{1}, w I_{2}\right): u \in a(d+g), v \in(a(e+h)+b(d+g)+b(e+h)\right. \\
& +b(f+i)+c(e+h)), w \in(a(f+i)+c(d+g)+c(f+i))\} .
\end{aligned}
$$

This shows that $x(y+z)=x y+x z$. Similarly, it can be shown that $(y+z) x=y x+z x$ and $\therefore$ is distributive over + . The proof is complete.

Lemma 2.8. Let $\left(X\left(I_{1}, I_{2}\right),+,.\right)$ be a refined neutrosophic hyperring. Then for all $x, y, z, w \in X\left(I_{1}, I_{2}\right)$, we have

(i) $-(-x)=x$

(ii) $-(x+y)=-x-y$.

(iii) $(x+y)(z+w)=x z+x w+y z+y w$.

Lemma 2.9. Let $A\left(I_{1}, I_{2}\right)$ be a nonempty subset of a refined neutrosophic hyperring $\left(X\left(I_{1}, I_{2}\right),+,.\right) . A\left(I_{1}, I_{2}\right)$ is a refined neutrosophic hyperideal of $X\left(I_{1}, I_{2}\right)$ if and only if:

(i) $x, y \in A\left(I_{1}, I_{2}\right)$ implies that $x-y \subseteq A\left(I_{1}, I_{2}\right)$, and

(ii) $x \in A\left(I_{1}, I_{2}\right)$ and $r \in X\left(I_{1}, I_{2}\right)$ imply that $x r, r x \in A\left(I_{1}, I_{2}\right)$.

Theorem 2.10. Let $A\left(I_{1}, I_{2}\right)$ and $B\left(I_{1}, I_{2}\right)$ be refined neutrosophic hyperideals of a refined neutrosophic hyperring $\left(X\left(I_{1}, I_{2}\right),+,.\right)$ and let $\left\{A\left(I_{1}, I_{2}\right)_{i}\right\}_{i=1}^{n}$ be a family of refined neutrosophic hyperideals of $\left(X\left(I_{1}, I_{2}\right),+,.\right)$. Then:

(i) $\bigcap_{i=1}^{n} A\left(I_{1}, I_{2}\right)_{i}$ is a refined neutrosophic hyperideal of $X\left(I_{1}, I_{2}\right)$.

(ii) $\oplus \sum_{i=1}^{n} A_{i}\left(I_{1}, I_{2}\right)$ is a refined neutrosophic hyperideal of $X\left(I_{1}, I_{2}\right)$.

(iii) $A\left(I_{1}, I_{2}\right)+B\left(I_{1}, I_{2}\right)$ is a refined neutrosophic hyperideal of $X\left(I_{1}, I_{2}\right)$.

(iv) $A\left(I_{1}, I_{2}\right)+B\left(I_{1}, I_{2}\right)$ is a refined neutrosophic hyperideal of $X\left(I_{1}, I_{2}\right)$.

Lemma 2.11. Let $A\left(I_{1}, I_{2}\right)$ be a refined neutrosophic hyperideal of a refined neutrosophic hyperring $\left(X\left(I_{1}, I_{2}\right),+,.\right)$. Then

(i) $A\left(I_{1}, I_{2}\right)+A\left(I_{1}, I_{2}\right)=A\left(I_{1}, I_{2}\right)$.

(ii) $x+A\left(I_{1}, I_{2}\right)=A\left(I_{1}, I_{2}\right)$ for all $x \in A\left(I_{1}, I_{2}\right)$. 
Proof. (i) Let $y=\left(p, q I_{1}, r I_{2}\right)$. Then

$$
\begin{aligned}
y \in & A\left(I_{1}, I_{2}\right)+A\left(I_{1}, I_{2}\right) \\
& \Leftrightarrow y \in u+u \text { for some } u=\left(a, b I_{1}, c I_{2}\right) \in A\left(I_{1}, I_{2}\right) \\
& \Leftrightarrow y \in A\left(I_{1}, I_{2}\right) \text { since } u+u \subseteq A\left(I_{1}, I_{2}\right) \\
& \therefore \quad A\left(I_{1}, I_{2}\right)+A\left(I_{1}, I_{2}\right)=A\left(I_{1}, I_{2}\right) .
\end{aligned}
$$

(ii) Let $y=\left(p, q I_{1}, r I_{2}\right)$. Then

$$
\begin{aligned}
y \in & x+A\left(I_{1}, I_{2}\right) \\
& \Leftrightarrow y \in x+u \text { for some } u=\left(a, b I_{1}, c I_{2}\right) \in A\left(I_{1}, I_{2}\right) \\
& \Leftrightarrow y \in A\left(I_{1}, I_{2}\right) \text { since } x+u \subseteq A\left(I_{1}, I_{2}\right)
\end{aligned}
$$

$\therefore x+A\left(I_{1}, I_{2}\right)=A\left(I_{1}, I_{2}\right)$.

Theorem 2.12. Let $A\left(I_{1}, I_{2}\right)$ be a refined neutrosophic hyperideal of a refined neutrosophic hyperring $\left(X\left(I_{1}, I_{2}\right),+,.\right)$. Then

(i) $\left(A\left(I_{1}, I_{2}\right)+x\right)+\left(A\left(I_{1}, I_{2}\right)+y\right)=A\left(I_{1}, I_{2}\right)+x+y$ for all $x, y \in X\left(I_{1}, I_{2}\right)$.

(ii) $A\left(I_{1}, I_{2}\right)+x=A\left(I_{1}, I_{2}\right)+y$ for all $y \in A\left(I_{1}, I_{2}\right)+x$.

(iii) $A\left(I_{1}, I_{2}\right)+x+y=A\left(I_{1}, I_{2}\right)+z$ if $z \in x+y$ for all $x, y, z \in X\left(I_{1}, I_{2}\right)$.

Proof. (i) Let $v=\left(p, q I_{1}, r I_{2}\right)$. Then

$$
\begin{aligned}
v \in & \left(A\left(I_{1}, I_{2}\right)+x\right)+\left(A\left(I_{1}, I_{2}\right)+y\right) \\
& \Leftrightarrow v \in(u+u)+x+y \text { for some } u=\left(a, b I_{1}, c I_{2}\right) \in A\left(I_{1}, I_{2}\right) \\
& \Leftrightarrow v \in A\left(I_{1}, I_{2}\right)+x+y \text { since } u+u \subseteq A\left(I_{1}, I_{2}\right) \\
& \therefore \quad\left(A\left(I_{1}, I_{2}\right)+x\right)+\left(A\left(I_{1}, I_{2}\right)+y\right)=A\left(I_{1}, I_{2}\right)+x+y .
\end{aligned}
$$

(ii) Suppose that $y \in A\left(I_{1}, I_{2}\right)+x$. Let $v=\left(p, q I_{1}, r I_{2}\right)$. Then

$$
\begin{aligned}
v \in & A\left(I_{1}, I_{2}\right)+x \\
& \Leftrightarrow v \in A\left(I_{1}, I_{2}\right)+A\left(I_{1}, I_{2}\right)+x \\
& \Leftrightarrow v \in A\left(I_{1}, I_{2}\right)+y \\
& \therefore \quad A\left(I_{1}, I_{2}\right)+x=A\left(I_{1}, I_{2}\right)+y .
\end{aligned}
$$

(iii) Suppose that $z \in x+y$ for all $x, y, z \in X\left(I_{1}, I_{2}\right)$. Let $v=\left(p, q I_{1}, r I_{2}\right)$. Then

$$
\begin{aligned}
v \in & A\left(I_{1}, I_{2}\right)+x+y \\
& \Leftrightarrow v \in u+(x+y) \text { for some } u=\left(a, b I_{1}, c I_{2}\right) \in A\left(I_{1}, I_{2}\right) \\
& \Leftrightarrow v \in u+z \\
& \Leftrightarrow v \in A\left(I_{1}, I_{2}\right)+z .
\end{aligned}
$$

$\therefore A\left(I_{1}, I_{2}\right)+x+y=A\left(I_{1}, I_{2}\right)+z$.

Theorem 2.13. Let $A\left(I_{1}, I_{2}\right)$ be a refined neutrosophic hyperideal of a refined neutrosophic hyperring $\left(X\left(I_{1}, I_{2}\right),+,.\right)$. Then $A\left(I_{1}, I_{2}\right)$ is normal in $X\left(I_{1}, I_{2}\right)$ only if $A$ is normal in $X$.

Proof. Suppose that $A$ is normal in $X$. Let $u=\left(x, y I_{1}, z I_{2}\right) \in X\left(I_{1}, I_{2}\right)$. Then there exists $v=\left(a, b I_{1}, c I_{2}\right) \in$ $A\left(I_{1}, I_{2}\right)$ such that

$$
\begin{aligned}
u+A\left(I_{1}, I_{2}\right)-u= & u+v-u \\
= & \left(x, y I_{1}, z I_{2}\right)+\left(a, b I_{1}, c I_{2}\right)-\left(x, y I_{1}, z I_{2}\right) \\
= & \left\{\left(p, q I_{1}, r I_{2}\right): p \in x+a-x, q \in y+b-y,\right. \\
& r \in z+c-z\} \\
= & \left\{\left(p, q I_{1}, r I_{2}\right): p \in x+A-x \subseteq A,\right. \\
& q \in y+A-y \subseteq A, r \in z+A-z \subseteq A\} \\
= & \left\{\left(p, q I_{1}, r I_{2}\right): p \in A, q \in A, r \in A\right\} \\
\subseteq & A\left(I_{1}, I_{2}\right)
\end{aligned}
$$

which shows that $A\left(I_{1}, I_{2}\right)$ is normal in $X\left(I_{1}, I_{2}\right)$. 
Example 2.14. Let $X_{1}\left(I_{1}, I_{2}\right)$ and $X_{2}\left(I_{1}, I_{2}\right)$ be two refined neutrosophic hyperrings and let $X\left(I_{1}, I_{2}\right)=$ $X_{1}\left(I_{1}, I_{2}\right) \oplus X_{2}\left(I_{1}, I_{2}\right)$ be their direct sum. Let $\phi_{i}: X\left(I_{1}, I_{2}\right) \rightarrow X_{i}\left(I_{1}, I_{2}\right)$ with $i=1,2$ be the projections defined by

$$
\phi_{1}(x, y)=x, \phi_{2}(x, y)=y \quad \forall(x, y) \in X\left(I_{1}, I_{2}\right) .
$$

Then $\phi_{i}$ are refined neutrosophic good(strong) homomorphisms.

Theorem 2.15. Let $\phi: X_{1}\left(I_{1}, I_{2}\right) \rightarrow X_{2}\left(I_{1}, I_{2}\right)$ be a refined neutrosophic good(strong) hyperring homomorphism from $\left(X_{1}\left(I_{1}, I_{2}\right),+,.\right)$ into $\left.X_{2}\left(I_{1}, I_{2}\right),+^{\prime}, .^{\prime}\right)$. Then

(i) $\phi\left(\left(0,0 I_{1}, 0 I_{2}\right)\right)=\left(0,0 I_{1}, 0 I_{2}\right)$.

(ii) $\phi(-x)=-\phi(x)$ for all $x \in X_{1}\left(I_{1}, I_{2}\right)$.

(iii) Ker $\phi$ is not a refined neutrosophic hyperideal of $X_{1}\left(I_{1}, I_{2}\right)$.

Proof. (i) and (ii) are clear.

(iii) It should be noted that only the element $\left(0,0 I_{1}, 0 I_{2}\right) \in X_{1}\left(I_{1}, I_{2}\right)$ is the only element in $\operatorname{Ker} \phi$. Since $\phi\left(I_{i}\right)=I_{i}$ for all $i=1,2$, it follows that no other element of $X_{1}\left(I_{1}, I_{2}\right)$ can be in the kernel of $\phi$. Hence, $\operatorname{Ker} \phi$ is not closed under - and consequently, it cannot be a refined neutrosophic hyperideal of $X_{1}\left(I_{1}, I_{2}\right)$.

However, it can be shown that $\operatorname{Ker} \phi$ is a subhyperring of $X_{1}\left(I_{1}, I_{2}\right)$ but not a refined neutrosophic

subhyperring of $X_{1}\left(I_{1}, I_{2}\right)$.

\section{Conclusion}

We have in this paper introduced the new concept of refined neutrosophic algebraic hyperstructures. We have studied refined neutrosophic Krasner hyperrings in particular. Their refined neutrosophic hypersubstructures were studied and their basic properties were presented. It was established that every refined neutrosophic Krasner hyperring is a Krasner hyperring. It was also established that the kernel of a refined neutrosophic Krasner hyperring homomorphism cannot be be a refined neutrosophic hyperideal but it can be a subhyperring. In our next paper to be titled Refined Neutrosophic Algebraic Hyperstructures II, we will study refined neutrosophic multiplicative hyperrings, their hypersubstructures and their properties.

\section{Appreciation}

The authors are grateful to all the anonymous reviewers for their useful observations, comments and suggestions.

\section{References}

[1] A.A.A. Agboola, On Refined Neutrosophic Algebraic Structures, Neutrosophic Sets and Systems, vol.10, pp. 99-101, 2015.

[2] A.A.A. Agboola, A.D. Akinola and O.Y. Oyebola, Neutrosophic Rings I, Int. J. of Math. Comb., vol. 4, pp. 1-14, 2011.

[3] A.A.A. Agboola, E.O. Adeleke and S.A. Akinleye, Neutrosophic Rings II, Int. J. of Math. Comb., vol. 2, pp. 1-8, 2012.

[4] A.A.A. Agboola, Akwu A.O. and Y.T. Oyebo, Neutrosophic Groups and Neutrosopic Subgroups, Int. J. of Math. Comb., vol. 3, pp. 1-9, 2012.

[5] A.A.A. Agboola and B. Davvaz, On Neutrosophic Canonical Hypergroups and Neutrosophic Hyperrings, Neutrosophic Sets and Systems, vol. 2, pp. 34-41, 2014.

[6] A.A.A. Agboola, B. Davvaz and F. Smarandache, Neutrosophic Quadruple Hyperstructures, Annals of Fuzzy Mathematics and Informatics, vol. 14, pp. 29-42, 2017.

[7] S.A. Akinleye, F. Smarandache and A.A.A. Agboola, On Neutrosophic Quadruple Algebraic Structures, Neutrosophic Sets and Systems, vol. 12, pp. 122-126, 2016. 
[8] M. Al-Tahan and B. Davvaz, Refined neutrosophic quadruple (po-)hypergroups and their fundamental group, Neutrosophic Sets and Systems, vol. 27, pp. 138-153, 2019.

[9] A. Asokkumar, Hyperlattice formed by the idempotents of a hyperring, Tamkang J. Math., vol. 38(3), pp. 209-215, 2007.

[10] A. Asokkumar and M. Veelrajan, Characterizations of regular hyperrings, Italian J. Pure and App. Math., vol. 22, pp. 115-124, 2007.

[11] A. Asokkumar and M. Veelrajan, Hyperrings of matrices over a regular hyperring, Italian J. Pure and App. Math., vol. 23, pp. 113-120, 2008.

[12] A.R. Bargi, A class of hyperrings, J. Disc. Math. Sc. and Cryp., vol. 6, pp. 227-233, 2003.

[13] T. Bera, S. Broumi and N.K. Mahapatra, Behaviour of ring ideal in neutrosophic and soft sense, Neutrosophic Sets and Systems, Vol. 25(1), pp. 1-24, 2019.

[14] T. Bera and N.K. Mahapatra, On Neutrosophic Soft Prime Ideal, Neutrosophic Sets and Systems, Vol. 20(6), pp. 54-75, 2018.

[15] P. Corsini, Prolegomena of Hypergroup Theory, Second edition, Aviain editore, 1993.

[16] P. Corsini and V. Leoreanu, Applications of Hyperstructure Theory, Advances in Mathematics, Kluwer Academic Publishers, Dordrecht, 2003.

[17] B. Davvaz, Polygroup Theory and Related Systems, World Sci. Publ., 2013.

[18] B. Davvaz, Isomorphism theorems of hyperrings, Indian J. Pure Appl. Math., vol. 35(3), pp. 321-333, 2004.

[19] B. Davvaz, Approximations in hyperrings, J. Mult.-Valued Logic Soft Comput., vol. 15(5-6), pp. 471488, 2009.

[20] B. Davvaz and A. Salasi, A realization of hyperrings, Comm. Algebra, vol. 34, pp. 4389-4400, 2006.

[21] B. Davvaz and T. Vougiouklis, Commutative rings obtained from hyperrings $\left(H_{v}\right.$-rings) with $\alpha^{*}$ relations, Comm. Algebra, vol. 35, pp. 3307-3320, 2007.

[22] B. Davvaz and V. Leoreanu-Fotea, Hyperring Theory and Applications, International Academic Press, USA, 2007.

[23] M. De Salvo, Hyperrings and hyperfields, Annales Scientifiques de l'Universite de Clermont-Ferrand II, vol. 22, pp. 89-107, 1984.

[24] E.O. Adeleke, A.A.A. Agboola and F. Smarandache, Refined Neutrosophic Rings I, International Journal of Neutrosophic Science (IJNS), vol. 2(2), pp. 77-81, 2020. (DOI:10.5281/zenodo.3728222)

[25] E.O. Adeleke, A.A.A. Agboola and F. Smarandache, Refined Neutrosophic Rings II, International Journal of Neutrosophic Science (IJNS), vol. 2(2), pp. 89-94, 2020. (DOI:10.5281/zenodo.3728235)

[26] F. Smarandache(2003), A Unifying Field in Logics: Neutrosophic Logic, Neutrosophy, Neutrosophic Set, Neutrosophic Probability, (3rd edition), American Research Press, Rehoboth, http://fs.gallup.unm.edu/eBook-Neutrosophic4.pdf.

[27] F. Smarandache, n-Valued Refined Neutrosophic Logic and Its Applications in Physics, Progress in Physics, USA, vol. 4, pp. 143-146, 2013.

[28] F. Smarandache, (T,I,F)- Neutrosophic Structures, Neutrosophic Sets and Systems, vol. 8, pp. 3-10, 2015.

[29] M.A. Ibrahim, A.A.A. Agboola, E.O. Adeleke and S.A. Akinleye, Introduction to Neutrosophic Subtraction Algebra and Neutrosophic Subtraction Semigroup, International Journal of Neutrosophic Science (IJNS), vol. 2(1), pp. 47-62, 2020. (DOI:10.5281/zenodo.3724603)

[30] K. Krasner, A class of hyperrings and hyperfields, Int. J. Math. and Math. Sci., vol. 6(2), pp. 307-311, 1983. 
[31] F. Marty, Sur une generalization de la notion de groupe, 8th Congress Math. Scandinaves, Stockholm, Sweden, pp. 45-49, 1934.

[32] S. Mirvakili, S.M. Anvariyeh and B. Davvaz, On $\alpha$-relation and transitivity conditions of $\alpha$, Comm. Algebra, vol. 36, pp. 1695-1703, 2008.

[33] S. Mirvakili and B. Davvaz, Applications of the $\alpha^{*}$-relation to Krasner hyperrings, J. Algebra, vol. 362, pp. 145-156, 2012.

[34] J. Mittas, Hypergroupes canoniques, Math. Balkanica, vol. 2, pp. 165-179, 1972.

[35] Muthusamy Velrajan and Arjunan Asokkumar, Note on Isomophism Theorems of Hyperrings, Int. J. Math. Math. Sc., Hindawi Publishing Corporation, pp. 1-12, 2010.

[36] A. Nakassis, Expository and survey article of recent results in hyperring and hyperfield theory, Internat. J. Math. Math. Sci., vol. 11, pp. 209-220, 1988.

[37] R. Rota, Strongly distributive multiplicative hyperrings, J. Geo., vol. 39(1-2), pp. 130-138, 1990.

[38] M. Ştefănescu, Constructions of hyperfields and hyperrings, Stud. Cercet. Ştiinţ. Ser. Mat. Univ. Bacău No., vol. 16, pp. 563-571, 2006.

[39] W.B. Vasantha Kandasamy and F. Smarandache, Neutrosophic Rings, Hexis, Phoenix, Arizona, 2006 http://fs.gallup.unm.edu/NeutrosophicRings.pdf

[40] T. Vougiouklis, Hyperstructures and their Representations, Hadronic Press, Inc, 115, Palm Harber, USA, 1994.

[41] T. Vougiouklis, The fundamental relation in hyperrings. The general hyperfield, Proc. Fourth Int. Congress on Algebraic Hyperstructures and Applications (AHA 1990), World Scientific, pp. 203-211, 1991. 